\title{
LHX6 acts as a novel potential tumour suppressor with epigenetic inactivation in lung cancer
}

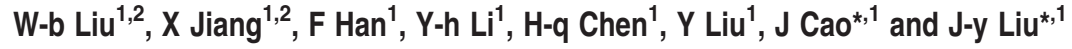

\begin{abstract}
LIM homeobox domain 6 (LHX6) is a putative transcriptional regulator that controls the differentiation and development of neural and lymphoid cells. However, the function of $L H X 6$ in cancer development remains largely unclear. Recently, we found that $L H X 6$ is hypermethylated in lung cancer. In this study, we analysed its epigenetic regulation, biological functions, and related molecular mechanisms in lung cancer. Methylation status was evaluated by methylation-specific PCR and bisulfite genomic sequencing. LHX6 mRNA levels were measured in relation to the methylation status. The effects of LHX6 expression on tumourigenesis were studied in vitro and in vivo. LHX6 was readily expressed in normal lung tissues without methylation, but was downregulated or silenced in lung cancer cell lines and tissues with hypermethylation status. Treatment of lung cancer cells with the demethylating agent 5-aza-2'-deoxycytidine restored LHX6 expression. Moreover, LHX6 hypermethylation was detected in $56 \%(52 / 93)$ of primary lung cancers compared with none (0/20) of the tested normal lung tissues. In lung cancer cell lines 95D and H358, forced expression of LHX6 suppressed cell viability, colony formation, and migration, induced apoptosis and G1/S arrest, and inhibited their tumorigenicity in nude mice. On the other hand, knockdown of LHX6 expression by RNA interference increased cell proliferation and inhibited apoptosis and cell cycle arrest. These effects were associated with upregulation of p21 and p53, and downregulation of Bcl-2, cyclinD1, c-myc, CD44, and MMP7. In conclusion, our results suggest that LHX6 is a putative tumour suppressor gene with epigenetic silencing in lung cancer.
\end{abstract}

Cell Death and Disease (2013) 4, e882; doi:10.1038/cddis.2013.366; published online 24 October 2013

Subject Category: Cancer

Lung cancer is one of the most common malignancies and the leading cause of cancer-related death worldwide. ${ }^{1}$ Although traditional cytology and imaging technology have important roles in the diagnosis of lung cancer, $67 \%$ of newly diagnosed lung cancer patients are still detected at an advanced stage. ${ }^{2}$ Effective early diagnosis and targeted therapies to reduce mortality would benefit from a better understanding of the key molecular changes in normal cells, which lead to precancerous lesions and malignant tumour cells.

Lung carcinogenesis is a multi-phase process involving the accumulation of multiple genetic and epigenetic changes. ${ }^{3-6}$ Alterations in normal DNA methylation patterns are fundamental to the tumorigenic process that contributes to all of the typical hallmarks of a cancer cell. ${ }^{7,8}$ Hypermethylation of tumour suppressor genes is associated with epigenetically mediated gene silencing. Recently, an increasing number of genes inactivated by $\mathrm{CpG}$ island hypermethylation have been recognised as important components involved in multistep cancer development in humans..$^{9-13}$ Characterisation of novel functional genes associated with promoter methylation may help us to understand the molecular mechanisms of alterations in tumour-suppressive pathways involved in lung carcinogenesis, and find better potential targets for the diagnosis and treatment of lung cancer.
The human LIM homeobox domain 6 (LHX6) gene is located on 9q33.2 and encodes a LIM homeodomain transcription factor involved in embryogenesis, more specifically in head development. ${ }^{14}$ LHX6 activity is required for normal tangential and radial migration of GABAergic interneurons and specification of neuronal subtypes in the neocortex and hippocampus. ${ }^{15} \mathrm{LHX} 6$ and $L H X 8$ co-ordinately induce neuronal expression of Sonic hedgehog that controls the generation of interneuron progenitors. ${ }^{16}$ In addition, $L H X 6$ hypermethylation acts as a sensitive methylation marker in head and neck carcinomas and has an important role in early diagnosis of cervical cancer. ${ }^{17-19}$ Recently, we identified LHX6 hypermethylation in lung cancer. However, the role of LHX6 in lung cancer remains unclear. In the present study, we investigated the epigenetic regulation and biological function of $L H X 6$ and its molecular basis in lung cancer.

\section{Results}

LHX6 is downregulated or inactivated in lung cancer tissues and cell lines. First, we performed reverse transcription-polymerase chain reaction (RT-PCR) to detect LHX6 mRNA expression in 10 primary lung cancer tissues and their corresponding adjacent non-tumour tissues.

\footnotetext{
${ }^{1}$ Institute of Toxicology, College of Preventive Medicine, Third Military Medical University, Chongqing 400038, China

${ }^{*}$ Corresponding author: J-y Liu or J Cao, Institute of Toxicology, College of Preventive Medicine, Third Military Medical University, 30 Gaotanyan Street, Shapingba District, Chongqing 400038, China. Tel: +862368752296 or +8623 68772533; Fax: +862368752296 or +8623 68772533; E-mail: jinyiliutmmu@163.com or caojia1962@126.com

${ }^{2}$ These authors contributed equally to this work.

Keywords: LHX6; DNA methylation; tumour suppressor; lung cancer; epigenetics

Abbreviations: 5-aza-dC, 5-aza-2'-deoxycytidine; LHX6, LIM Homeobox domain 6; BGS, bisulfite genomic sequencing; MSP, methylation-specific polymerase chain reaction; RT-PCR, reverse transcription-polymerase chain reaction

Received 24.6.13; revised 07.8.13; accepted 07.8.13; Edited by A Stephanou
} 
As shown in Figure 1a, LHX6 expression was downregulated in lung cancer tissues compared with that in the adjacent normal tissues. Next, we examined $L H X 6$ expression in nine lung cancer cell lines and the normal human bronchial epithelial (HBE) cell line by RT-PCR and quantitative RT$\mathrm{PCR}$. As shown in Figure $1 \mathrm{~b}$ and Supplementary Figure S1, $L H X 6$ expression was reduced or silenced in eight (90\%) cell lines, but was readily detected in $\mathrm{H} 1395$ cells and the normal cell line HBE. These results suggest aberrant gene silencing of $L H X 6$ in lung cancer.

LHX6 hypermethylation is associated with transcriptional silencing. To clarify whether expression of the LHX6 gene was regulated by DNA hypermethylation, we first analysed the methylation status of $L H X 6$ in human cell lines by methylation-specific polymerase chain reaction (MSP). MSP region information is shown in Supplementary Figure S2a.
The reliability of the MSP results was verified by DNA sequencing (Supplementary Figure S2b). As shown in Figure 1c, we found that all lung cancer cell lines in our study showed hypermethylation, whereas HBE cells exhibited an unmethylated status. However, the methylation pattern was heterogeneous in the CpG island region. Fully methylated $L H X 6$ was detected in cell lines A549, 95D, and $\mathrm{H} 1975$, whereas $L H X 6$ was partly methylated in SPC-A-1, $\mathrm{H} 358, \mathrm{H} 1650$, LTEP, H1395, and $\mathrm{H} 460$ cell lines, which showed downregulation of $L H X 6$ expression. The methylation status was inversely correlated with the LHX6 expression level in all lung cancer cell lines and HBE cells except in H1395 cells. These results suggest that LHX6 hypermethylation is associated with transcriptional downregulation or silencing.

Next, we detected the $L H X 6$ methylation status in 93 cases of human primary lung cancer and 20 cases of normal

a

$\begin{array}{lllllllllllllll}\text { MW } & \mathrm{N} 1 & \mathrm{~N} 2 & \mathrm{~N} 3 & \mathrm{~N} 4 & \mathrm{~N} 5 & \mathrm{~N} 6 & \mathrm{~T} 1 & \mathrm{~T} 2 & \mathrm{~T} 3 & \mathrm{~T} 4 & \mathrm{~T} 5 & \mathrm{~T} 6 & \mathrm{NC} & \mathrm{PC}\end{array}$

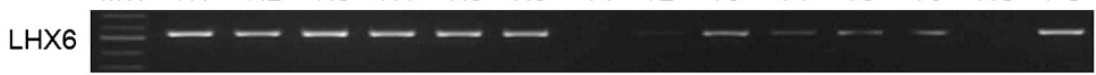

$\beta-\operatorname{actin} \equiv---\infty-\infty-\infty-$

b

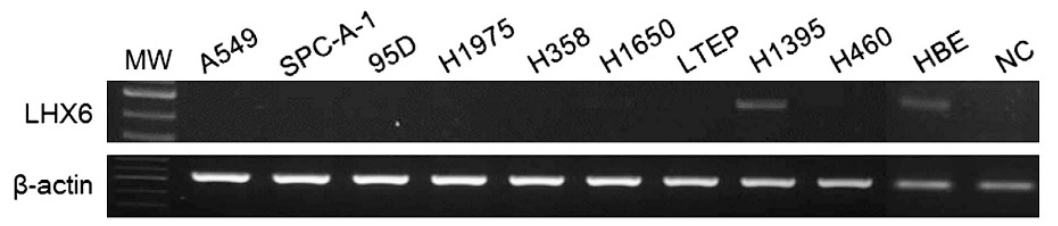

C

$\mathrm{MW} \frac{\mathrm{N} 1}{\mathrm{U} M} \frac{\mathrm{N} 2}{\mathrm{U} M} \frac{\mathrm{N} 3}{U \mathrm{M}} \frac{\mathrm{N} 4}{\mathrm{U} M} \frac{\mathrm{T} 111}{U \mathrm{M}} \frac{\mathrm{T} 118}{U \mathrm{M}} \frac{\mathrm{T} 122}{\mathrm{U} M} \frac{\mathrm{T} 127}{U \mathrm{M}} \frac{\mathrm{T} 133}{\mathrm{U} M}$

LHX6

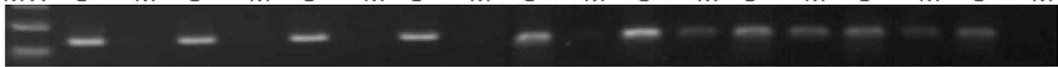

SPC-A-1 $\frac{\mathrm{H} 358}{M} \frac{\mathrm{H} 1650}{M} \frac{\text { LTEP }}{M} \frac{\mathrm{H} 1395}{M} \frac{\mathrm{H} 460}{M} \quad$ A549 $\quad$ 95D $\frac{\mathrm{H} 1975}{M} \quad$ HBE

LHX6 MW $\overline{U M} \overline{U M} \frac{\mathrm{U} M}{U M} \bar{U} \mathrm{U}$

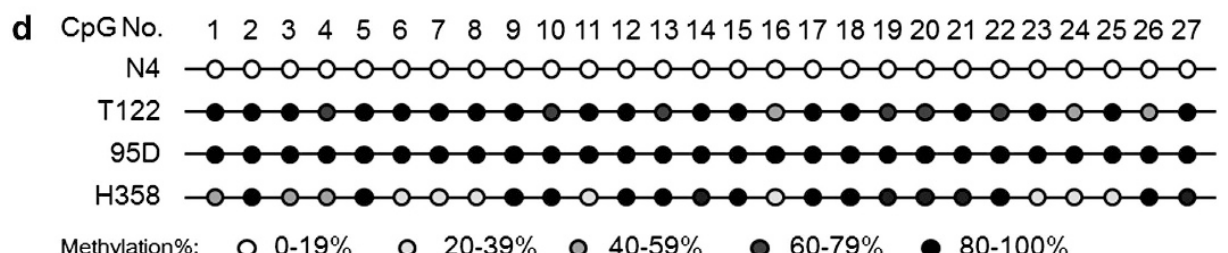

$\mathbf{e}$

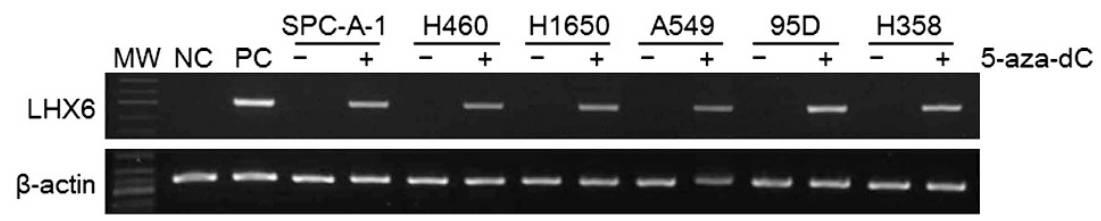

Figure 1 Epigenetic silencing of $L H X 6$ in lung cancer tissues and cell lines. (a) Representative results of $L H X 6 \mathrm{mRNA}$ expression in tissues. $L H X 6 \mathrm{mRNA}$ expression was significantly decreased in primary lung cancer tissues compared with that in the corresponding normal tissues as detected by RT-PCR. (b) mRNA expression of LHX6 in lung cancer cell lines was determined by semi-quantitative RT-PCR with $\beta$-actin as the internal control. LHX6 was weakly or not expressed in all lung cancer cell lines except for H1395. (c) Representative MSP results of the LHX6 methylation status in human tissues and cell lines. LHX6 was unmethylated in human normal lung tissues, but frequently hypermethylated in human lung cancer tissues and cell lines. MW, molecular weight; $\mathrm{M}$, methylated product; $\mathrm{U}$, unmethylated product; $\mathrm{N}$, normal tissue; T, lung cancer tissue. (d) BGS analysis confirmed the methylation status of LHX6 in human tissues and cell lines. LHX6 was fully methylated in 95D cells, partially methylated in H358 and T122 cells, and unmethylated in normal tissue. (e) LHX6 mRNA expression was restored in lung cancer cell lines after treatment with the demethylation agent 5-aza-dC. '-' indicates DMSO control; and ' + ' indicates 5-aza-dC 
lung tissue. Using MSP, we found LHX6 hypermethylation in 52 (including 43 samples with partial methylation and 9 samples with fully methylation) out of $93(56 \%)$ lung cancer samples and no $L H X 6$ hypermethylation $(0 / 20)$ in normal lung tissues (representative results are shown in Figure 1c). Next, we analysed the association of the $L H X 6$ methylation status and clinicopathological characteristics in 62 patients with lung cancer. However, as shown in Supplementary Table S1, there was no correlation between LHX6 methylation and clinicopathological features such as age, gender, histological type, pathological stage, or differentiation status.

We performed bisulfite genomic sequencing (BGS) to provide a detailed map of the DNA methylation pattern within the $\mathrm{CpG}$ island region of the $L H X 6$ gene. The size of the PCR product was $324 \mathrm{bp}$ and included the transcriptional start site (-99 to +225 bp; Supplementary Figure S2a). Representative results are shown in Figure 1d. In $27 \mathrm{CpG}$ sites of this region, $L H X 6$ was fully hypermethylated in 95D cells. In H358 cells and lung cancer tissue T122, LHX6 was partially methylated. As expected, LHX6 was unmethylated in HBE cells expressing $L H X 6$. These results are in agreement with those of MSP, and suggest that DNA methylation may be responsible for loss of $L H X 6$ expression.

5-aza-2'-deoxycytidine restores $L H X 6$ expression. To further test the hypothesis that loss of $L H X 6$ expression is caused by hypermethylation of the $L H X 6$ gene, lung cancer cell lines were treated with the demethylating agent 5-aza2'-deoxycytidine (5-aza-dC). Representative results of semi-quantitative RT-PCR and quantitative RT-PCR analyses are illustrated in Figure 1e and Supplementary Figure S3, respectively. As expected, lung cancer cell lines treated with 5-aza-dC, which initially showed high levels of $L H X 6$ methylation, were induced to express $L H X 6$. Quantitative RT-PCR analyses showed that the ratio of $L H X 6$ to $\beta$-actin transcripts was increased several fold in the 5-aza-dCtreated group compared with that in the control group. These results strongly indicated that hypermethylation is responsible for defective expression of $L H X 6$ in lung cancer.

LHX6 inhibits cell growth in vitro and in vivo. To evaluate the functional significance of LHX6 in lung cancer, we examined growth suppression by overexpression of $L H X 6$ in 95D and $H 358$ cells that showed loss of $L H X 6$ expression and $\mathrm{CpG}$ island hypermethylation. The transfection efficiency is shown in Supplementary Figure S4. Re-expression of LHX6 in these cell lines was evidenced by RT-PCR and western blot analyses (Figure $2 \mathrm{a}$ ). The results of the cell proliferation assay indicated that ectopic expression of $L H X 6$ significantly inhibited the growth of 95D and H358 cells compared with that in the control groups (Figure $2 b$ ). To confirm the inhibitory effect of LHX6 on the growth of lung cancer cell lines, we used a colony formation assay using 95D and H358 cells. Compared with control vectortransfected cells without LHX6 expression, we found a greatly reduced number of colonies formed by $\mathrm{LHX6}$ transfected 95D and $\mathrm{H} 358$ cells $(P<0.01$; Figure $2 \mathrm{c})$. These data further demonstrated that $L H X 6$ inhibits lung cancer cell proliferation.
We confirmed the effect of $L H X 6$ on cell proliferation by examining $L H X 6$ overexpression in a tumourigenesis assay using nude mice. Tumour growth of H358 cells overexpressing LHX6 was significantly reduced compared with that of control cells (Figure 2d). The mean tumour weight was significantly less in nude mice injected with $\mathrm{LHX6}$ overexpressing $\mathrm{H} 358$ cells compared with that in mice injected with control vector-transfected cells $(P<0.01$; Figure $2 \mathrm{~d}$ ). These results suggest that $L H X 6$ is a tumour suppressor gene that negatively regulates tumour growth both in vitro and in vivo.

LHX6 induces apoptosis and cell cycle arrest. We next determined whether inhibition of tumour cell growth by LHX6 is related to apoptosis and cell cycle arrest. First, we performed an apoptosis assay by flow cytometry (Figure 3a). We found that ectopic expression of LHX6 caused a significant increase in early apoptosis and late apoptosis compared with that of control vector-transfected 95D (Figure 3a(1)) and H358 (Figure 3a(2)) cells. In addition, morphological observation and Hoechst 33258 staining were performed to definitively establish the effect of LHX6 expression on apoptosis (Supplementary Figure S5).

Next, the effect of LHX6 expression on the cell cycle was evaluated by flow cytometry (Figure 3b). Among 95D cells without exogenous $L H X 6$ expression, the percentages of cells in $\mathrm{G} 1, \mathrm{~S}$, and $\mathrm{G} 2$ phases were $43.26 \pm 1.10,40.39 \pm 0.44$, and $16.35 \pm 0.66 \%$, respectively. In contrast, among 95D cells with re-expression of $L H X 6$, the percentages of cells in $\mathrm{G} 1, \mathrm{~S}$, and G2 phases were $51.96 \pm 1.00,34.86 \pm 0.52$, and $13.18 \pm 0.48 \%$, respectively (Figure $3 b(1)$ ). This trend was also found in $\mathrm{H} 358$ cells. The percentages of $\mathrm{H} 358$ cells in $\mathrm{G} 1$, $\mathrm{S}$, and $\mathrm{G} 2$ phases were $52.71 \pm 0.47,32.95 \pm 1.22$, and $14.35 \pm 0.74 \%$, respectively. In contrast, among H358 cells with re-expression of $L H X 6$, the percentages of cells in $\mathrm{G} 1, \mathrm{~S}$, and $\mathrm{G} 2$ phases were $62.48 \pm 1.19,26.70 \pm 0.76$, and $10.83 \pm 0.43 \%$, respectively (Figure $3 b(2)$ ). These results demonstrate a reduction in the percentages of cells in $S$ and G2 phases, and retention of 95D and H358 cells with restoration of $L H X 6$ expression in $\mathrm{G} 1$ phase $(P<0.05)$.

LHX6 expression induces cell migration. The effect of LHX6 expression on lung cancer cell motility was investigated by a wound healing assay. LHX6 transfectants spread along wound edges much slower than that of control vector transfectants (Figure 4). Quantitative analyses at $48 \mathrm{~h}$ confirmed a significant reduction in wound closure by LHX6-transfected 95D (Figure 4a) and H358 (Figure 4b) cells compared with that of empty vector-transfected control cells.

Knockdown of LHX6 promotes cell proliferation. To confirm the inhibitory effect of $L H X 6$ expression on cell growth, we knocked down LHX6 expression in the LHX6expressing cell line HBE by siRNA. Real-time quantitative RT-PCR showed that LHX6 expression was significantly decreased by $L H X 6$ siRNA (Figure $5 \mathrm{a}$ ). We found that knockdown of $L H X 6$ promoted the growth of HBE cells by a CCK-8 cell viability assay (Figure $5 b$ ). Accordingly, knockdown of $L H X 6$ significantly increased colony formation by 
a1
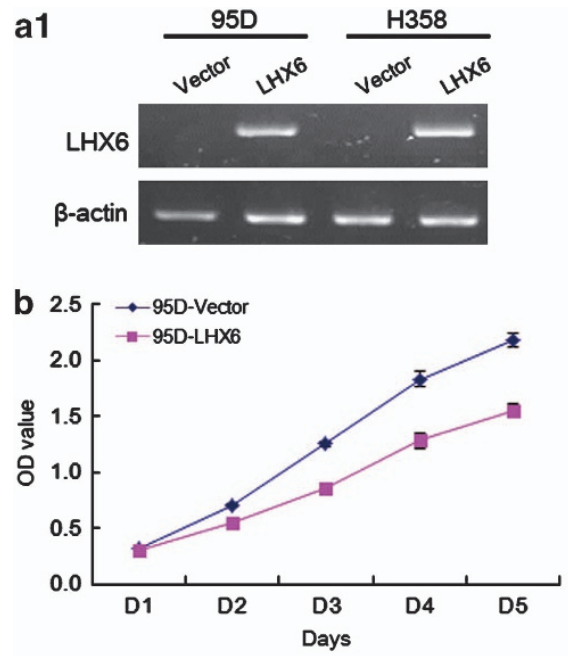

c

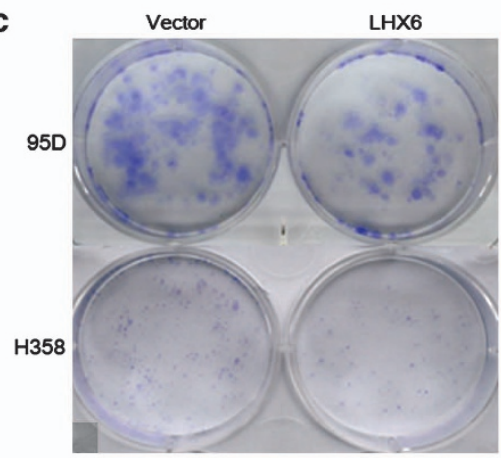

d

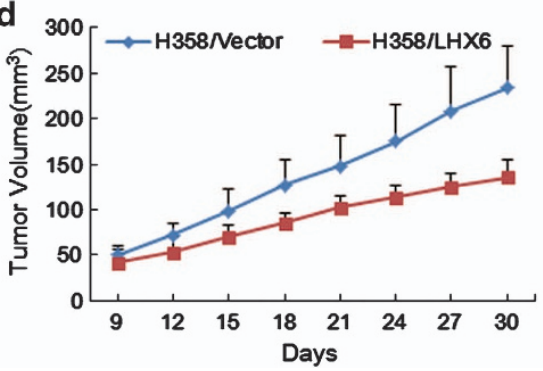

a2
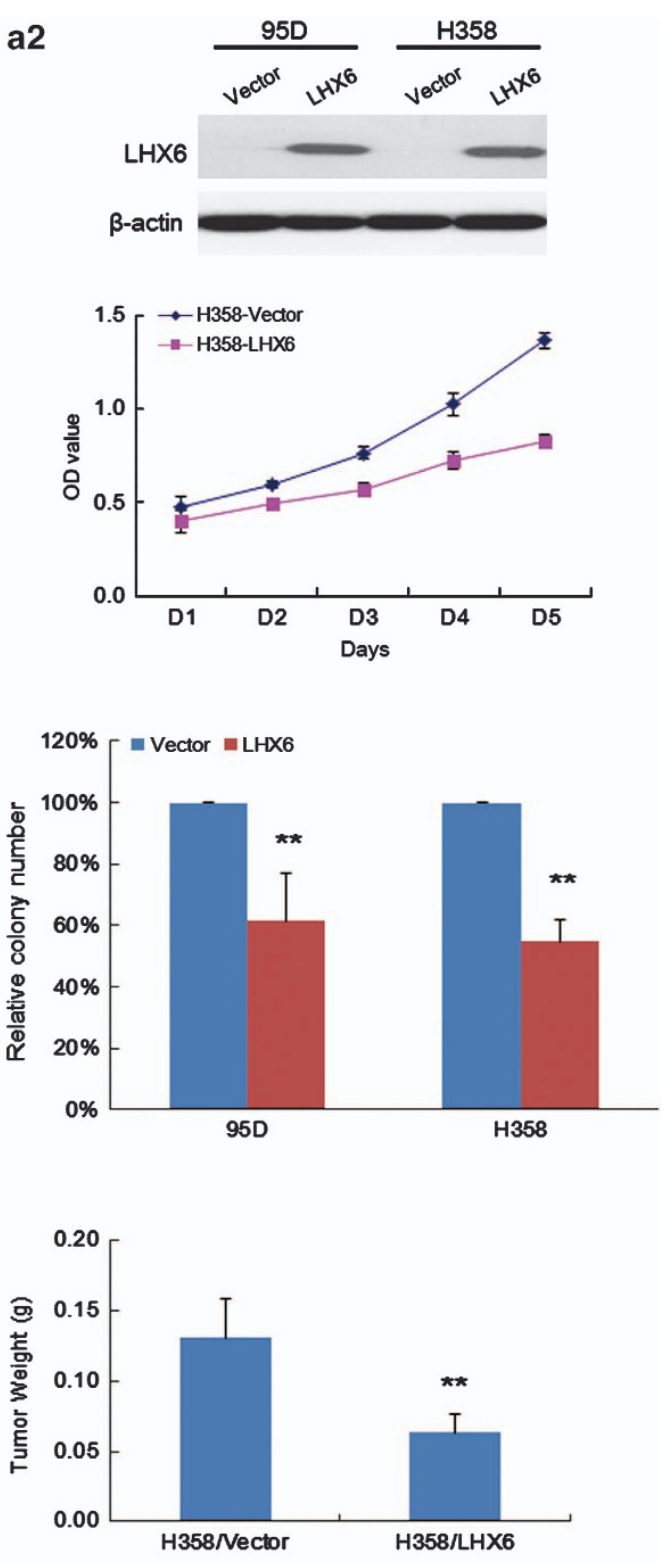

Figure 2 LHX6 inhibits cell growth. (a) Re-expression of LHX6 mRNA and protein in transfected cell lines 95D and H358 was confirmed by RT-PCR (a1) and western blotting (a2), respectively. (b) LHX6 significantly inhibited the proliferation of 95D and H358 cells as shown by CCK-8. These data are shown as the mean \pm S.D. of three independent experiments. (c) Colony formation assays were performed to confirm the effect of LHX6 on cell growth. Quantitative analysis of colony numbers is shown in the right panel. Compared with empty vector transfectants, a significant reduction of colony numbers was observed for LHX6 transfectants. (d) Inhibition of tumour growth by LHX6 expression in vivo. Growth curves of tumours in nude mice showed that $L H X 6$ transfectants grew slower than empty vector transfectants. ${ }^{* *} P<0.01$

more than $60 \%$ compared with that of cells transfected with control siRNA (Figure 5c). These results further indicated that $L H X 6$ acts as a potential tumour suppressor gene in lung cancer.

Next, we further examined the effect of $L H X 6$ knockdown on cell cycle regulation. Among HBE cells transfected with negative control siRNA, $70.92 \pm 2.37 \%$ were in G1 phase, $19.18 \pm 1.76 \%$ were in $S$ phase, and $9.91 \pm 0.61 \%$ were in G2 phase. In contrast, among LHX6 siRNA-transfected HBE cells, $61.65 \pm 1.99 \%$ were in $\mathrm{G} 1$ phase, $21.57 \pm 0.85 \%$ were in $S$ phase, and $16.79 \pm 1.14 \%$ were in G2 phase $(P<0.05)$ (Figure 5d). Thus, LHX6-targeted siRNA decreased the number of cells in $\mathrm{G} 1$ phase, whereas significantly increasing the number of cells in $\mathrm{G} 2$ phase, suggesting that loss of $L H X 6$ expression increases cellular proliferation.

Finally, we examined the effect of LHX6 knockdown on apoptosis by flow cytometry. Our results showed that both early apoptotic cells $(7.26 \pm 1.05 \%$ versus $4.96 \pm 0.63 \%)$ and late apoptotic cells $(23.00 \pm 1.41 \%$ versus $19.75 \pm 1.63 \%)$ among LHX6 siRNA-transfected HBE cells decreased significantly compared with those among control siRNAtransfected HBE cells $(P<0.05)$ (Figure $5 e$ ).

Molecular targets of LHX6 in cell lines. To gain insights into the molecular mechanisms of the tumour-suppressive effect of LHX6, LHX6-modulated downstream target genes 

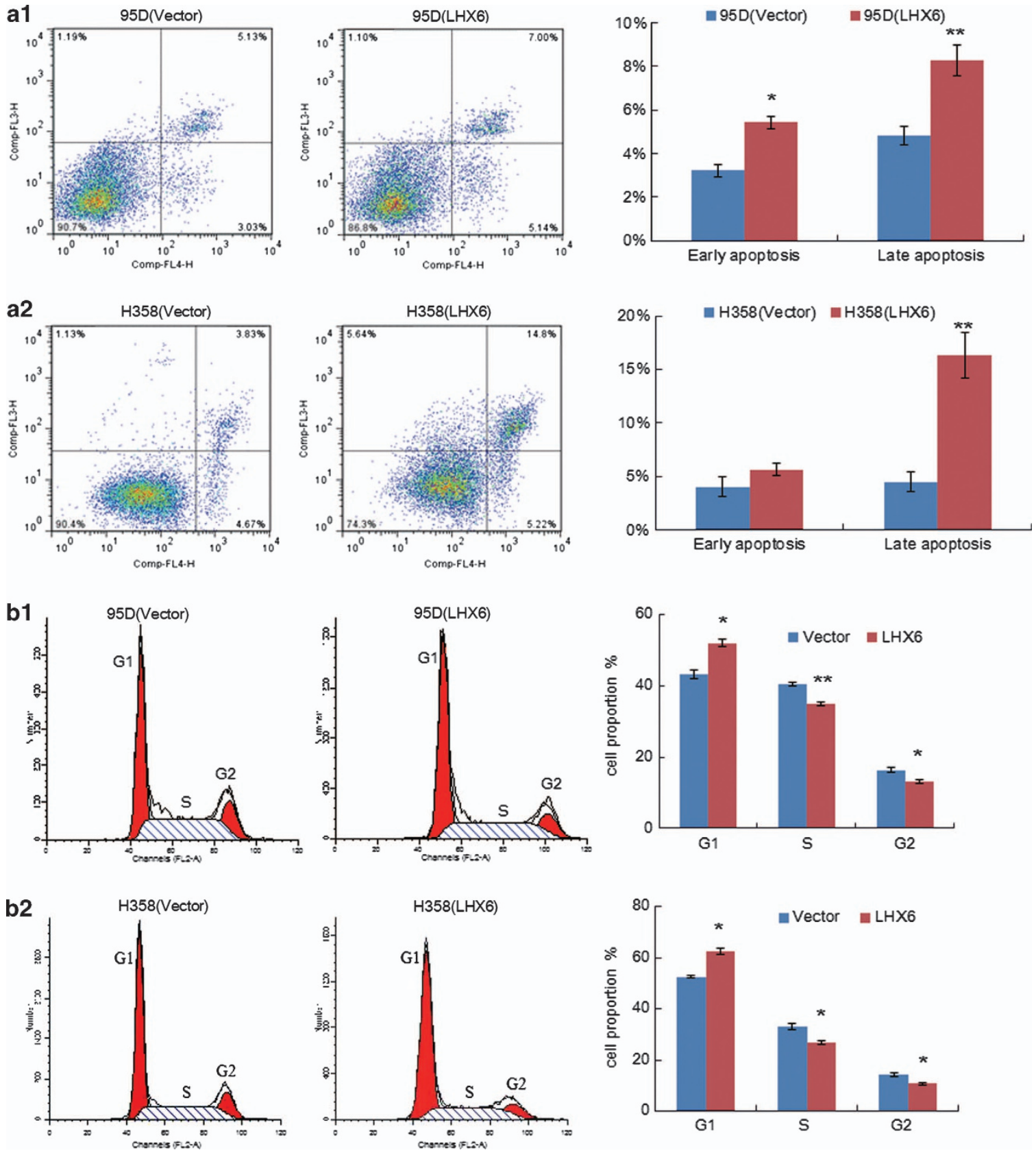

Figure 3 Effect of LHX6 expression on apoptosis and the cell cycle. (a) Flow cytometric assays with Annexin V-APC and 7-AAD double staining of 95D (a1) and H358 (a2) cells. Left panel shows representative dots plots and right panels show the quantitative analysis. (b) Cell cycle analysis by flow cytometry. The distribution of empty or LHX6 expression vector-transfected 95D (b1) and H358 (b2) cells in cell cycle phases. The percentages are represented by bar graphs. Each experiment was repeated three times. ${ }^{*} P<0.05 ;{ }^{*} P<0.01$

were characterised by quantitative RT-PCR analysis of H358 cells stably transfected with $L H X 6$. As shown in Figure 6a, LHX6 increased expression of the pro-apoptotic gene p53 and downregulated the anti-apoptotic gene Bcl-2. LHX6 also enhanced expression of $p 21$, a cell cycle regulator. LHX6 exerted an anti-proliferative effect by downregulating expression of cell proliferation genes cyclinD1 and $c$-myc, which are both important G1 phase regulators. Moreover, inhibition of cell migration by LHX6 resulted from downregulation of $C D 44$ and MMP7 expression. On the other hand, loss of $L H X 6$ expression had the opposite effect on regulation of these genes (Figure 6b). The results suggest that LHX6 acts as tumour suppressor by regulating the expression of important genes involved in proliferation, apoptosis, and migration pathways (Figure 6c).

\section{Discussion}

Several lines of evidence support the hypothesis that epigenetic changes by DNA methylation have an important role in lung carcinogenesis. ${ }^{20-23}$ Recently, epigenetic silencing of many novel genes that function as putative tumour 
a

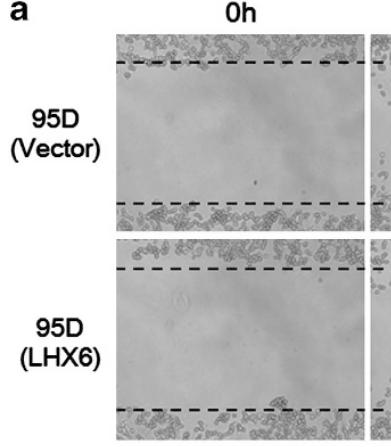

b

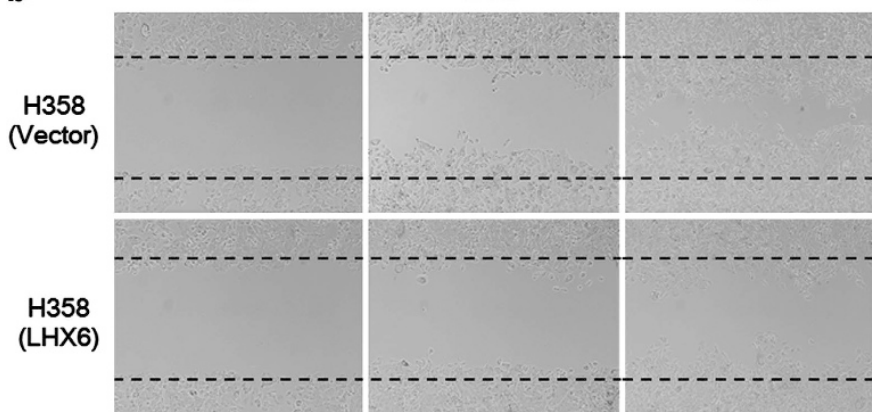

$24 h$

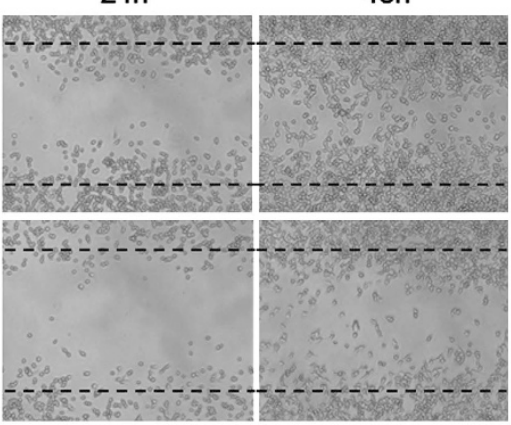

$24 h$

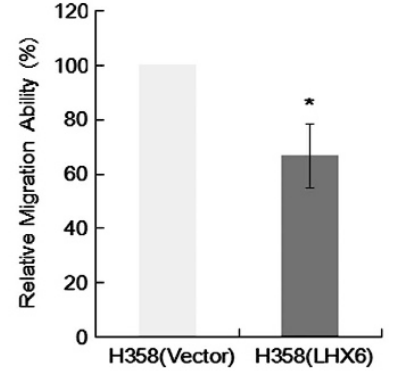

Figure 4 Cell motility as determined by a wound healing assay. The ability of LHX6 transfectants to spread along wound edges in 95D (a) and H358 (b) cells was significantly reduced compared with that of control cells. Quantification of the migrated cells is shown in the right panel. All data represent the mean \pm S.D. of three independent experiments. ${ }^{*} P<0.05$

suppressor genes has been reported to contribute to human lung cancer. ${ }^{9-11,21,23}$ In this study, we identified a typical CpG island located in the transcription region of the $L H X 6$ gene, which showed a hypermethylation status in lung cancer. To our knowledge, this is the first study of epigenetic regulation of $L H X 6$ expression and its function in lung cancer.

In this study, we found that $L H X 6$ hypermethylation is frequent and cancer specific in lung cancer. Our results suggest that LHX6 methylation is associated with tumorigenesis. In accordance with our study, methylation levels of the $L H X 6$ promoter are also strongly associated with cervical carcinogenesis and cancer development. ${ }^{18,19}$ This epigenetic alteration of the $L H X 6$ gene begins at a relatively early stage, suggesting its potential as a biomarker for early diagnosis and prevention of head and neck carcinomas, and cervical cancer. ${ }^{17,18}$

We also found that the reduction of $L H X 6$ expression was related to $\mathrm{CpG}$ island methylation of $L H X 6$. Furthermore, a high dose of 5-aza-dC stimulated $L H X 6$ re-expression in most of the lung cancer cell lines, suggesting that $\mathrm{CpG}$ island methylation is the predominant regulatory mechanism of LHX6 inactivation in lung cancer. However, compared with 5-aza-dC alone, exposure to various concentrations of 5-aza$\mathrm{dC}$ with trichostatin A increases $L H X 6$ expression more significantly in cervical cell lines. ${ }^{18,19}$ Thus, other epigenetic modifications such as histone modifications may be involved in silencing $L H X 6$ expression in lung cancer. Accordingly, studies on the human $L H X 3$ gene have shown that cellspecific expression of two different transcripts of $L H X 3$ is driven by epigenetic and genetic regulatory systems. ${ }^{24}$ Considering that $L H X 3$ and $L H X 6$ belong to the same family, it is plausible to assume that human $L H X 6$ expression may be regulated in a similar manner. In the future, the mechanism of $L H X 6$ regulation requires further study.

LHX6 is a putative transcriptional regulator that controls the differentiation and development of neural and lymphoid cells, particularly in the central nervous system. ${ }^{14,15}$ In addition to its established role in muscle development, little is known about the function of LHX6, especially in cancer. Recently, several other $L H X$ genes have also been implicated in tumourigenesis. ${ }^{25-29}$ LHX4 may act as a tumour suppressor by downregulation of alpha-fetoprotein expression in hepatocarcinogenesis. ${ }^{25}$ Inactivation of $L H X 8$ expression reduces the transcription of pro-apoptotic genes bax, caspase-2, and caspase-3 in mouse oocytes. ${ }^{26}$ Epigenetic alteration of the LHX9 gene is involved in glioma cell invasiveness and migration. ${ }^{27}$ Hypermethylation-mediated reduction of $L M X 1 A$ expression inhibits tumourigenesis, epithelial-to-mesenchymal transition, and stem-like properties of the epithelia in gastric and ovarian cancers. ${ }^{28,29}$ In addition, several novel epigenetically silenced genes have been found to suppress cell growth in numerous types of cancer. ${ }^{30-35}$ These studies have suggested that LHX6 may have a potential tumour suppressor role in lung cancer. ${ }^{25-35}$

Thus, the tumour suppressor role of LHX6 in lung cancer was investigated both in vitro and in vivo. Ectopic expression of LHX6 in the silenced 95D and H358 cell lines showed a significant growth suppressive effect by inhibition of cell proliferation and colony formation. A decrease of tumour growth by LHX6 was confirmed by the reduction of tumourigenesis in nude mice. On the other hand, siRNA-mediated knockdown of $L H X 6$ in HBE cells resulted in significantly 
a

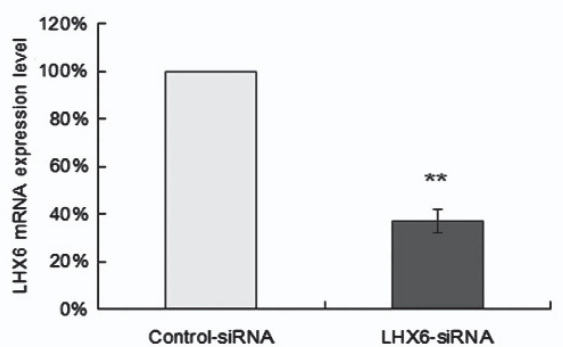

c1

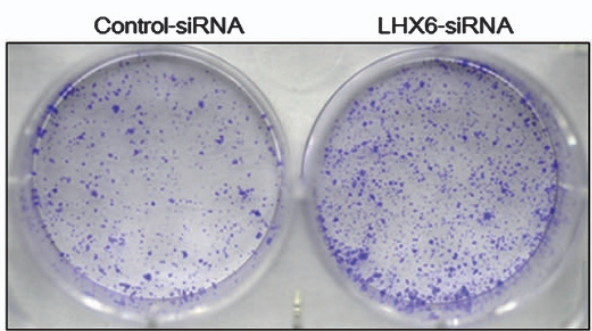

d
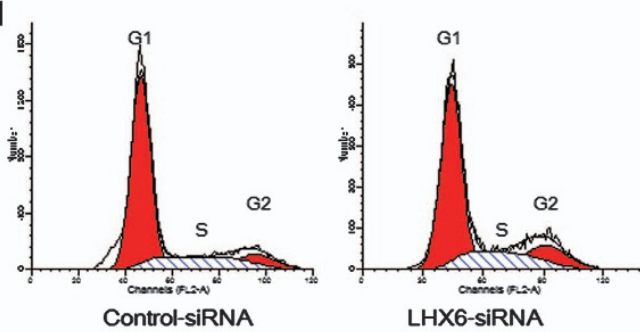

LHX6-siRNA

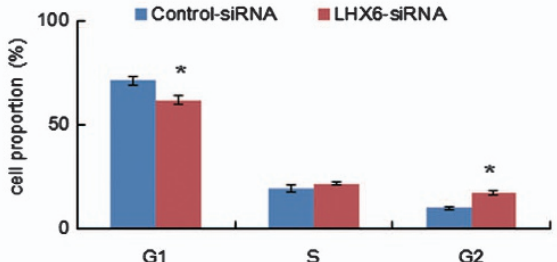

b

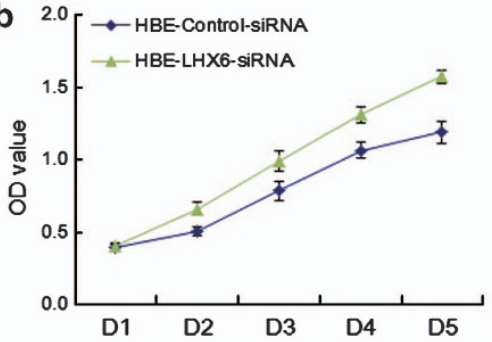

c2

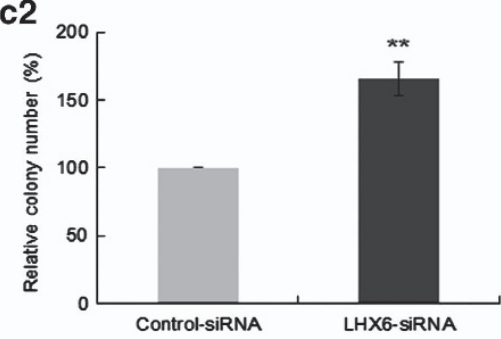

e
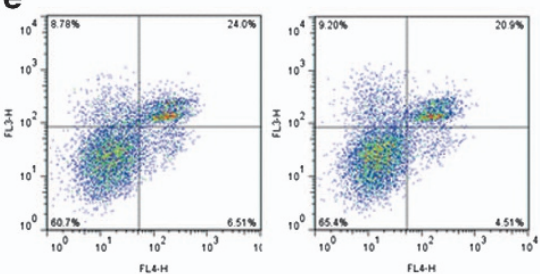

Control-siRNA

LHX6-siRNA

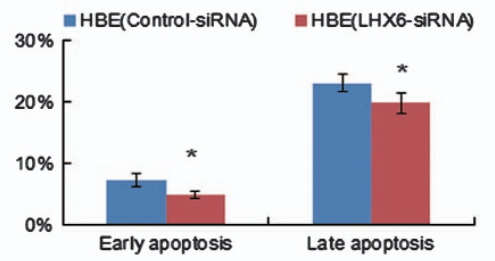

Figure 5 Effect of $L H X 6$ knockdown on cell growth. (a) LHX6 mRNA-targeting siRNA and a non-targeting control siRNA were transfected into $L H X 6$-expressing HBE cells. The efficiency of LHX6 knockdown was examined by real-time RT-PCR. (b) siRNA-mediated knockdown of LHX6 induced cell proliferation as shown by CCK-8. (c) Colony formation assays confirmed the effect of LHX6 expression on cell growth. Colony numbers were increased by knockdown of $L H X 6$. Colonies were photographed under a phase-contrast microscope (left, c1). Average numbers of colonies are represented by the bar graph (right, c2). Each experiment was repeated three times. (d) Cell cycle analysis by flow cytometry. The distribution of siRNA negative control- and LHX6 siRNA-transfected HBE cells in cell cycle phases. The percentages are represented by the bar graph. Each experiment was repeated three times. (e) The effect of LHX6 knockdown on apoptosis was analysed by flow cytometry. ${ }^{*} P<0.05$; ${ }^{* *} P<0.01$

increased cell proliferation. These results indicate that LHX6 functions as a tumour suppressor in lung carcinogenesis. Similar to our findings, overexpression of $L H X 6$ in cervical cancer cells causes a reduction of cell proliferation and suppresses the tumorigenic phenotype, further demonstrating the growth suppressive effects of $L H X 6$ expression and suggesting that it may be a novel tumour suppressor gene in cervical cancer. ${ }^{19}$

LHX6 is a LIM homeobox transcription factor expressed during embryogenesis. However, the molecular mechanisms that regulate $L H X 6$ transcriptional activity are unknown. Previously, LHX6 and an interaction with PITX2 were found to regulate cell proliferation in the cervical loop and promote cell differentiation in the anterior region of the incisor. ${ }^{36}$ Recently, Zhang et al. ${ }^{37}$ demonstrated that LHX6 directly interacts with PITX2 to inhibit PITX2 transcriptional activity and activation of multiple promoters. PITX2 directly interacts with $\beta$-catenin to synergistically regulate $L E F-1$ expression, which regulates the activity of several growth control genes. $^{38,39}$ These findings demonstrate new molecular mechanisms of LHX6 for normal craniofacial and tooth development. In our study, we also found that LHX6 decreased the expression of Wnt signalling target genes cyclinD1, c-myc, CD44, and MMP7. Our results suggest that LHX6 may be an important negative regulator of the WNT signalling pathway that is involved in many biological processes including embryogenesis and carcinogenesis. ${ }^{40}$ However, further definition and investigation of LHX6regulated genes are critical to understand the pathways through which LHX6 exerts its tumour-suppressive activity.

In conclusion, we have identified $L H X 6$ as a novel functional tumour suppressor gene that is silenced by CpG island 

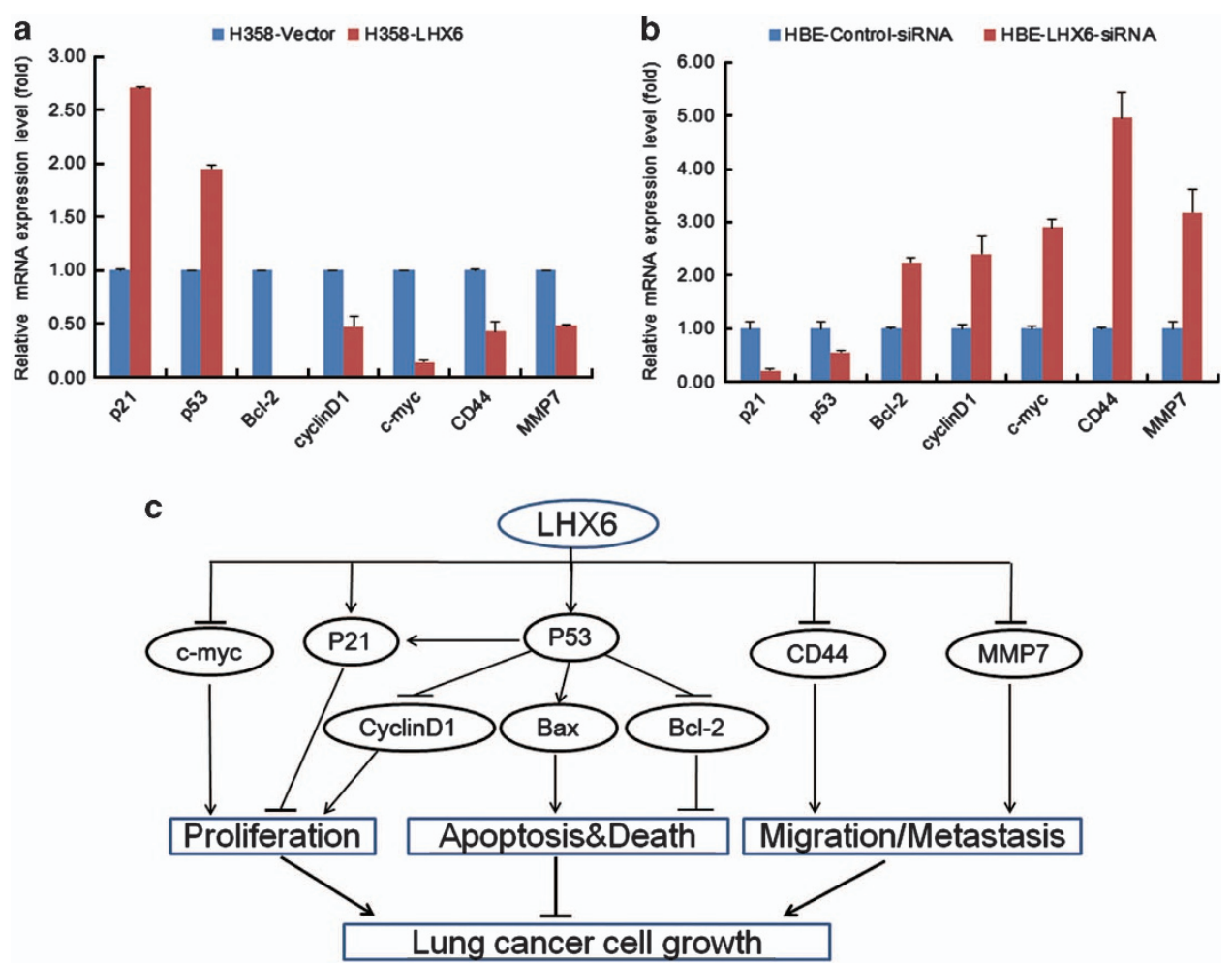

Figure 6 Effect of LHX6 on its downstream genes in cancer pathways. (a) Ectopic expression of LHX6 enhanced the expression of p21 and p53, and decreased the expression of Bcl-2, cyclinD1, c-myc, CD44, and MMP7. (b) Expression of p21 and p53 decreased, whereas the expression of Bcl-2, cyclinD1, c-myc, CD44, and MMP7 increased in HBE cells with knockdown of LHX6. (c) Schematic diagram for the mechanisms of LHX6 as a tumour suppressor

methylation in lung cancer. LHX6 contributes to the suppression of tumourigenesis by decreasing cell proliferation and inducing apoptosis and cell cycle arrest by regulating several important signalling pathways. LHX6 methylation may serve as a potential epigenetic biomarker for early diagnosis of patients with lung cancer.

\section{Materials and Methods}

Cell lines and tissue samples. Lung cancer cell lines (A549, SPC-A-1, 95D, H1975, H358, H1650, LTEP, H1395, and H460) and an immortalised HBE cell line were obtained from the American Type Culture Collection (Manassas, VA, USA) and Cell Biology Institute of Chinese Academy of Science (Shanghai, China). All cell lines were cultured in RPMI-1640 medium (Gibco BRL, Rockville, MD, USA) supplemented with $10 \%$ foetal bovine serum (Gibco BRL) and incubated in a humidified atmosphere with $5 \% \mathrm{CO}_{2}$ at $37^{\circ} \mathrm{C}$.

A total of 93 primary lung cancer tissues and 20 normal lung tissues were obtained during biopsy before any therapeutic intervention at the Affiliated Xi'nan Hospital of the Third Military Medical University. The tumours were classified according to the WHO histological typing of lung tumours, and staged following the TNM classification of malignant tumours defined by the International Union against Cancer. All experiments and procedures were approved by the Clinical Research Ethics Committee of the Third Military Medical University.

RNA extraction, RT-PCR, and real-time quantitative RT-PCR analyses. Total RNA was extracted from cells and tissues with Trizol (Invitrogen, Carlsbad, CA, USA) according to the manufacturer's protocol. cDNA was synthesised from $1 \mu \mathrm{g}$ total RNA using a PrimeScript RT reagent Kit with gDNA Eraser (Takara, Otsu, Japan). RT-PCR and real-time quantitative RT-PCR analyses were performed as described previously with the housekeeping gene $\beta$-actin used as an internal control. ${ }^{4-43}$ Primer sequences are listed in Supplementary Table S2.
DNA extraction, MSP, and BGS. Genomic DNA from cell lines and tissues was isolated by a DNA extraction kit (Promega, Madison, WI, USA) and chemically modified using an EZ DNA Methylation-Gold Kit (Zymo Research, Orange, CA, USA) according to the manufacturer's instructions. Primer pairs used for MSP and BGS are listed in Supplementary Table S2. For MSP, the protocol was carried out as reported previously. ${ }^{44-46}$ For BGS, the PCR products were purified and inserted into a pGEM-T easy vector (Promega). Ten colonies were chosen randomly for sequencing.

Demethylation agent 5 -aza-dC treatment. Demethylation was performed as reported previously. ${ }^{41}$ Briefly, lung cancer cells were seeded at a density of $1 \times 10^{6}$ cells per $\mathrm{ml}$ in $10-\mathrm{cm}$ dishes. After $24 \mathrm{~h}$ of culture, cells were treated with $10 \mu \mathrm{M}$ 5-aza-dC (Sigma, St. Louis, MO, USA) for 3 days. Control cells were treated in parallel with DMSO for each time point. The medium was replaced every day. Cells were harvested and mRNA expression of LHX6 was analysed by RT-PCR and quantitative RT-PCR.

Construction of the LHX6 overexpression vector. CDNA corresponding to the full-length human LHX6 gene was verified by sequencing and subcloned into the mammalian expression vector pIRES2-EGFP (Invitrogen). Lung cancer cell lines were transfected using X-treme Gene HP DNA transfection reagent (Roche, Mannheim, Germany) according to the manufacturer's instructions. Clones with stable transfection of LHX6 or empty vector were established with G418 (Invitrogen) selection for 2-3 weeks. Gene and protein expression of LHX6 was confirmed by RT-PCR and western blot analyses.

Knockdown of LHX6 by siRNA. Four siRNA sequences and a negative control sequence of the siRNA annealing sequence were designed, synthesised, and then subcloned into pcDNA6.2 GW/EmGFP siRNA vectors (Invitrogen). The HBE cell line with $L H X 6$ expression was transfected with the vectors carrying $L H X 6$ or negative control siRNAs. Knockdown efficiency was evaluated at $48 \mathrm{~h}$ after transfection by realtime quantitative RT-PCR. The siRNA with the highest knockdown efficiency was used for functional studies. Stably transfected cells with LHX6 knockdown were selected with $0.6 \mathrm{mg} / \mathrm{ml}$ Blasticidin S HCl (Invitrogen) for colony formation assays. 
Cell viability assay. Briefly, $8 \times 10^{3}$ cells per well were seeded in 96-well plates and transiently transfected with pIRES2-EGFP-LHX6, LHX6 siRNA, or control vectors. After 1-5 days of transfection, cell viability was evaluated by Cell Counting Kit-8 (CCK-8; Dojindo, Kumamoto, Japan) according to the manufacturer's instructions. Data were expressed as the optical density measured at a wavelength of $450 \mathrm{~nm}$. Experiments were carried out in triplicate.

Colony formation assay. For the overexpression assay, 95D and H358 cells $\left(1 \times 10^{5}\right.$ cells/well) were seeded in 24-well plates and transfected with pIRES2-EGFP-LHX6 or empty vectors. For the knockdown assay, HBE cells were transfected with $L H X 6$ siRNA or control vectors. At 2 days post transfection, cells were passaged at a 1:20 ratio into six-well plates containing medium with G418 or Blasticidin S HCl. After culturing for 14-21 days, cells were fixed with $4 \%$ paraformaldehyde and then stained with crystal violet. Colonies with more than 50 cells per colony were counted. All experiments were conducted three times in triplicates.

Hoechst 33258 staining. Hoechst 33258 staining was carried out at $48 \mathrm{~h}$ post transfection. The cells were fixed with $4 \%$ paraformaldehyde for $15 \mathrm{~min}$, washed with PBS, and then stained with Hoechst 33258 (Beyotime, Jiangsu, China) for $5 \mathrm{~min}$. After two washes with PBS, morphological changes of apoptotic nuclei were observed under a fluorescence microscope with excitation and emission at 346 and $460 \mathrm{~nm}$, respectively.

Flow cytometry. Cells with overexpression or knockdown of LHX6 were fixed in $70 \%$ ethanol and stained with $15 \mu \mathrm{g} / \mathrm{ml}$ propidium iodide at $4{ }^{\circ} \mathrm{C}$ in the dark. The cells were then analysed by a FACSCalibur (BD Biosciences, Franklin Lakes, NJ, USA). Cell cycle profiles were determined using ModFitLT software (Becton Dickinson, San Diego, CA, USA). Apoptosis was determined by dual staining with an Annexin V-APC and 7-AAD Apoptosis Detection kit (Keygen, Nanjing, China) according to the manufacturer's instructions, and then analysed by FlowJo software (TreeStar, San Carlos, CA, USA).

Wound healing assay. Cell migration was assessed by a scratch wound assay. Briefly, 95D and H358 cells $\left(5 \times 10^{5}\right.$ cells/well) stably transfected with pIRES2-EGFP-LHX6 or empty vector were cultured in six-well plates. At $90 \%$ confluence, three scratch wounds across each well were made using a P-200 pipette tip. At 0,24 and $48 \mathrm{~h}$ of culture, images were taken of the wound closure areas. The experiment was performed in triplicate wells and repeated three times.

In vivo tumorigenicity. $\mathrm{H} 358$ cells $\left(5 \times 10^{6}\right.$ cells in $\left.0.2 \mathrm{ml} \mathrm{PBS}\right)$ stably transfected with pIRES2-EGFP-LHX6 or empty vector were injected subcutaneously into the dorsal flank of 4-week-old female Balb/c nude mice $(n=4$ per group). Tumour volume $\left(\mathrm{mm}^{3}\right)$ was estimated by measuring the longest (a) and shortest (b) diameter of the tumour and calculated $a s b^{2} / 2$. All experimental procedures were approved by the Animal Ethics Committee of the Third Military Medical University.

Western blot analysis. Total protein was extracted from cell lines, and the protein concentration was measured by the Bradford method (Bio-Rad Laboratories, Hercules, CA, USA). Protein samples $(40 \mu \mathrm{g})$ were separated by $10 \%$ SDS-PAGE and then transferred to an equilibrated polyvinylidene difluoride membrane (Amersham Biosciences, Buckinghamshire, UK). After incubation at $4{ }^{\circ} \mathrm{C}$ overnight with a primary antibody against LHX6 (Santa Cruz Biotechnology, Santa Cruz, CA, USA) and then reacting with the secondary antibody, the proteins were detected by enhanced chemiluminescence (Amersham Corporation, Arlington Heights, IL, USA).

Statistical analysis. Statistical analyses were performed with SPSS 13.0 software (SPSS, Chicago, IL, USA). Data were expressed as the mean \pm S.D. Results were evaluated using the $t$-test, Fisher's exact test, and Mann-Whitney $U$-test. A $P$-value $<0.05$ was considered significant.

\section{Conflict of Interest}

The authors declare no conflict of interest.

Acknowledgements. This work was supported by grants from the National Natural Science Foundation of China $(81202238,81030052,81071695)$, Natural
Science Foundation Project of CQ CSTC of China (cstc2011jA10098), and the Third Military Medical University (2011XQN07). We thank YJ Li for technical assistance. The current study does not have any commercial connection with any other people or organisation.

1. Siegel R, Naishadham D, Jemal A. Cancer statistics, 2013. CA Cancer J Clin 2013; 63 : 11-30.

2. Mascaux C, Peled N, Garg K, Kato Y, Wynes MW, Hirsch FR. Early detection and screening of lung cancer. Expert Rev Mol Diagn 2010; 10: 799-815.

3. Breuer RH, Pasic A, Smit EF, van Vliet E, Vonk Noordegraaf A, Risse EJ et al. The natural course of preneoplastic lesions in bronchial epithelium. Clin Cancer Res 2005; 11: $537-543$

4. Thorgeirsson TE, Geller F, Sulem P, Rafnar T, Wiste A, Magnusson KP et al. A variant associated with nicotine dependence, lung cancer and peripheral arterial disease. Nature 2008; 452: 638-642.

5. Esteller M. Cancer epigenomics: DNA methylomes and histone-modification maps. Nat Rev Genet 2007; 8: 286-298.

6. Risch A, Plass C. Lung cancer epigenetics and genetics. Int J Cancer 2008; 123: 1-7.

7. Feil R, Fraga MF. Epigenetics and the environment: emerging patterns and implications. Nat Rev Genet 2012; 13: 97-109.

8. Rodríguez-Paredes M, Esteller M. Cancer epigenetics reaches mainstream oncology. Nat Med 2011; 17: 330-339.

9. Cui T, Chen Y, Yang L, Knösel T, Huber O, Pacyna-Gengelbach M et al. The p53 target gene desmocollin 3 acts as a novel tumor suppressor through inhibiting EGFR/ERK pathway in human lung cancer. Carcinogenesis 2012; 33: 2326-2333.

10. Jia $Y$, Yang $Y$, Brock MV, Zhan Q, Herman JG, Guo M. Epigenetic regulation of DACT2, A key component of the Wnt signaling pathway in human lung cancer. J Pathol 2013; 230: 194-204.

11. Yang L, Chen Y, Cui T, Knösel T, Zhang Q, Albring KF et al. Desmoplakin acts as a tumor suppressor by inhibition of the Wnt/ $\beta$-catenin signaling pathway in human lung cancer. Carcinogenesis 2012; 33: 1863-1870.

12. Liu W, Li X, Chu ES, Go MY, Xu L, Zhao G et al. Paired box gene 5 is a novel tumor suppressor in hepatocellular carcinoma through interaction with p53 signaling pathway. Hepatology 2011; 53: 843-853.

13. Xu L, Li X, Chu ES, Zhao G, Go MY, Tao $Q$ et al. Epigenetic inactivation of BCL6B, a novel functional tumour suppressor for gastric cancer, is associated with poor survival of gastric cancer. Gut 2012; 61: 977-985.

14. Grigoriou M, Tucker AS, Sharpe PT, Pachnis V. Expression and regulation of Lhx6 and Lhx7, a novel subfamily of LIM homeodomain encoding genes, suggests a role in mammalian head development. Development 1998; 125: 2063-2074.

15. Liodis P, Denaxa M, Grigoriou M, Akufo-Addo C, Yanagawa Y, Pachnis V. Lhx6 activity is required for the normal migration and specification of cortical interneuron subtypes. J Neurosci 2007; 27: 3078-3089.

16. Flandin $\mathrm{P}$, Zhao Y, Vogt D, Jeong J, Long J, Potter $\mathrm{G}$ et al. Lhx6 and Lhx8 coordinately induce neuronal expression of $S$ hh that controls the generation of interneuron progenitors. Neuron 2011; 70: 939-950.

17. Estécio MR, Youssef EM, Rahal P, Fukuyama EE, Góis-Filho JF, Maniglia JV et al. LHX6 is a sensitive methylation marker in head and neck carcinomas. Oncogene 2006; 25: 5018-5026.

18. Jung S, Jeong D, Kim J, Yi L, Koo K, Lee J et al. The role of hLHX6-HMR as a methylation biomarker for early diagnosis of cervical cancer. Oncol Rep 2010; 23: 1675-1682.

19. Jung S, Jeong D, Kim J, Yi L, Koo K, Lee J et al. Epigenetic regulation of the potential tumor suppressor gene, hLHX6.1, in human cervical cancer. Int J Oncol 2011; 38: 859-869.

20. Esteller M. Epigenetics in cancer. N Engl J Med 2008; 358: 1148-1159.

21. Okamoto J, Hirata T, Chen Z, Zhou HM, Mikami I, Li H et al. EMX2 is epigenetically silenced and suppresses growth in human lung cancer. Oncogene 2010; 29: 5969-5975.

22. Liu $Y, A n Q$, Li L, Zhang D, Huang J, Feng $X$ et al. Hypermethylation of p16INK4a in Chinese lung cancer patients: biological and clinical implications. Carcinogenesis 2003; 24: 1897-1901.

23. Tessema M, Klinge DM, Yingling CM, Do K, Van Neste L, Belinsky SA. Re-expression of CXCL14, a common target for epigenetic silencing in lung cancer, induces tumor necrosis. Oncogene 2010; 29: 5159-5170.

24. Yaden BC, MIII Garcia, Smith TP, Rhodes SJ. Two promoters mediate transcription from the human LHX3 gene: involvement of nuclear factor I and specificity protein 1 . Endocrinology 2006; 147: 324-337.

25. Hung TM, Hu RH, Ho CM, Chiu YL, Lee JL, Jeng YM et al. Downregulation of alphafetoprotein expression by LHX4: a critical role in hepatocarcinogenesis. Carcinogenesis 2011; 32: 1815-1823.

26. Choi Y, Ballow DJ, Xin Y, Rajkovic A. Lim homeobox gene, Ihx8, is essential for mouse oocyte differentiation and survival. Biol Reprod 2008; 79: 442-449.

27. Vladimirova V, Mikeska T, Waha A, Soerensen N, Xu J, Reynolds PC et al. Aberrant methylation and reduced expression of LHX9 in malignant gliomas of childhood. Neoplasia 2009; 11: 700-711.

28. Dong W, Feng $L$, Xie $Y$, Zhang $H$, Wu Y. Hypermethylation-mediated reduction of LMX1A expression in gastric cancer. Cancer Sci 2011; 102: 361-366. 
29. Chao TK, Yo YT, Liao YP, Wang YC, Su PH, Huang TS et al. LIM-homeobox transcription factor 1, alpha (LMX1A) inhibits tumourigenesis, epithelial-mesenchymal transition and stem-like properties of epithelial ovarian cancer. Gynecol Oncol 2013; 128: 475-482.

30. Melotte V, Lentjes MH, van den Bosch SM, Hellebrekers DM, de Hoon JP, Wouters KA et al. N-Myc downstream-regulated gene 4 (NDRG4): a candidate tumor suppressor gene and potential biomarker for colorectal cancer. J Natl Cancer Inst 2009; 101: 916-927.

31. Yu J, Ma X, Cheung KF, Li X, Tian L, Wang S et al. Epigenetic inactivation of T-box transcription factor 5, a novel tumor suppressor gene, is associated with colon cancer. Oncogene 2010; 29: 6464-6474.

32. Das PM, Thor AD, Edgerton SM, Barry SK, Chen DF, Jones FE. Reactivation of epigenetically silenced HER4/ERBB4 results in apoptosis of breast tumor cells. Oncogene 2010; 29: 5214-5219.

33. Du W, Wang S, Zhou Q, Li X, Chu J, Chang Z et al. ADAMTS9 is a functional tumor suppressor through inhibiting AKT/mTOR pathway and associated with poor survival in gastric cancer. Oncogene 2013; 32: 3319-3328.

34. Cheung KF, Lam CN, Wu K, Ng EK, Chong WW, Cheng AS et al. Characterization of the gene structure, functional significance, and clinical application of RNF180, a novel gene in gastric cancer. Cancer 2012; 118: 947-959.

35. Xu X, Liu RF, Wan BB, Xing WM, Huang J, Han ZG. Expression of a novel gene FAM43B repressing cell proliferation is regulated by DNA methylation in hepatocellular carcinoma cell lines. Mol Cell Biochem 2011; 354: 11-20.

36. Kioussi C, Briata P, Baek SH, Rose DW, Hamblet NS, Herman T et al. Identification of a Wnt $/ \beta$-Catenin $\rightarrow$ Pitx2 pathway mediating cell-type-specific proliferation during development. Cell 2011; 111: 673-685.

37. Zhang Z, Gutierrez D, Li X, Bidlack F, Cao H, Wang $\mathrm{J}$ et al. The LIM Homeodomain Transcription Factor LHX6: a transcriptional repressor that interacts with Pituitary homeobox 2 (PITX2) to regulate odontogenesis. J Biol Chem 2013; 288: 2485-2500.

38. Vadlamudi U, Espinoza HM, Ganga M, Martin DM, Liu X, Engelhardt JF et al. PITX2, betacatenin and LEF-1 interact to synergistically regulate the LEF-1 promoter. J Cell Sci 2000; 118: $1129-1137$.
39. Baek SH, Kioussi C, Briata P, Wang D, Nguyen HD, Ohgi KA et al. Regulated subset of G1 growth-control genes in response to derepression by the Wnt pathway. Proc Natl Acad Sci USA 2003; 100: 3245-3250.

40. Polakis P. Wnt signaling and cancer. Genes Dev 2000; 14: 1837-1851.

41. Liu WB, Han F, Jiang X, Yang LJ, Li YH, Liu Y et al. ANKRD18A as a novel epigenetic regulation gene in lung cancer. Biochem Biophys Res Commun 2012; 429: 180-185.

42. Liu WB, Liu JY, Ao L, Zhou ZY, Zhou YH, Cui ZH et al. Dynamic changes in DNA methylation during multistep rat lung carcinogenesis induced by 3-methylcholanthrene and diethylnitrosamine. Toxicol Lett 2009; 189: 5-13.

43. Liu WB, Liu JY, Ao L, Zhou ZY, Zhou YH, Cui ZH et al. Epigenetic silencing of cell cycle regulatory genes during 3-methylcholanthrene and diethylnitrosamine induced multistep rat lung cancer. Mol Carcinog 2010; 49: 556-565.

44. Liu WB, Ao L, Zhou ZY, Cui ZH, Zhou YH, Yuan XY et al. CpG island hypermethylation of multiple tumor suppressor genes associated with loss of their protein expression during rat lung carcinogenesis induced by 3-methylcholanthrene and diethylnitrosamine. Biochem Biophys Res Commun 2010; 402: 507-514.

45. Liu WB, Ao L, Cui ZH, Zhou ZY, Zhou YH, Yuan XY et al. Molecular analysis of DNA repair gene methylation and protein expression during chemical-induced rat lung carcinogenesis. Biochem Biophys Res Commun 2011; 408: 596-601.

46. Liu WB, Cui ZH, Ao L, Zhou ZY, Zhou YH, Yuan XY et al. Aberrant methylation accounts for cell adhesion-related gene silencing during 3-methylcholanthrene and diethylnitrosamine induced multistep rat lung carcinogenesis associated with overexpression of DNA methyltransferases 1 and 3a. Toxicol Appl Pharmacol 2011; 251: 70-78.

(c) $(\rightarrow)$ Cell Death and Disease is an open-access journal published by Nature Publishing Group. This work is licensed under a Creative Commons Attribution-NonCommercialNoDerivs 3.0 Unported License. To view a copy of this license, visit http://creativecommons.org/licenses/by-nc-nd/3.0/

Supplementary Information accompanies this paper on Cell Death and Disease website (http://www.nature.com/cddis) 